Revista Posgrado y Sociedad

Sistema de Estudios de Posgrado

Universidad Estatal a Distancia

ISSN 2215-2172

Costa Rica

revistaposgradoysoci@uned.ac.cr

\title{
La Transversalidad de Género en la Educación Superior: propuesta de un modelo de implementación
}

Gender mainstreaming in higher education: an implementation model

\author{
María Martha Durán \\ Escuela de Ciencias de la Administración \\ Universidad Estatal a Distancia
}

Volumen 12, Número 1

Marzo 2012

pp. $23-43$

Recibido: Junio, 2011

Aprobado: Setiembre, 2011 


\section{Resumen}

Se discute en este artículo sobre el origen y concepto de Transversalidad de Género, y su importancia en la Educación Superior. Se realiza una propuesta de modelo de implementación y se puntualiza en el área académica, sus fortalezas y limitaciones.

Palabras claves: Transversalidad de Género, Educación Superior, área académica, estrategias de Implementación.

\section{Abstract}

The article discusses the origin and concept of Gender Mainstreaming as well as its importance in Higher Education. A proposal for a model regarding its implementation is made and its strengths and limitations in the academic area are highlighted.

Keywords: Gender Mainstreaming, Higher Education, implementation strategies. 


\section{Introducción: El contexto actual de la educación}

Desde finales del siglo pasado, el sistema educativo se encuentra inmerso en un vertiginoso proceso de cambio, enmarcado en la globalización y el desarrollo científico en general, y en específico por el conjunto de transformaciones propiciadas por el desarrollo de las tecnologías de la información y de la comunicación, que dan origen a características particulares en las generaciones del nuevo siglo.

El actual contexto de cambio acelerado, complejo y retador, ha impulsado la creciente comprensión de la complejidad del ser humano y el abordaje de la Educación en sus múltiples funciones y contradicciones ${ }^{1}$. La Educación es objeto de una gran presión social, política y cultural para su adecuación al contexto porque, en tanto sistema abierto, la acción del sistema educativo está en constante relación con el entorno del cual recibe influencias y sobre el que, al mismo tiempo, influye.

Además es fundamental señalar que los sistemas educativos nacionales en el contexto actual, son responsabilidad irrenunciable de los gobiernos. De esta forma, el aspecto político es un elemento clave en relación con el tema educativo.

De esta forma, se asume el sistema educativo como dinámico y cambiante, lo que implica la posibilidad de modificar o reconstruir estereotipos a partir de metodologías innovadoras que

\footnotetext{
1 Potenciadora de progreso, bienestar, democracia, pero también agente que "refuerza, mantiene, produce y reproduce estereotipos de género" (Tomé, 1999: 175). Es uno de los espacios en donde se encuentra el germen de la discriminación y desigualdad.
}

permitan, como Acker (1995, p.75) propone, "la abolición del género como realidad cultural opresiva”, posibilitando la apertura de espacios para la reflexión y el cambio de normas y procedimientos formales $\mathrm{e}$ informales, así como en la subjetividad de las personas y el análisis de las representaciones sociales.

En la búsqueda de la articulación de igualdad y equidad (integrando sexo y género) y la ruptura de las asimetrías que históricamente hemos vivido, el enfoque de género como categoría de análisis es un instrumento conceptual que permite abordarlas, $y$ ha posibilitado el desarrollo de diferentes estrategias para promover la equidad de género.

Desde el siglo pasado diferentes movimientos feministas y mujeres en todo el mundo han impulsado $\mathrm{y}$ realizado esfuerzos que van desde la focalización en actividades específicas para las mujeres, pasando por el incremento de su participación en las agendas de desarrollo, hasta la estrategia de transversalidad de género (Gender Mainstreaming) cuyo impacto estructural se refleja en los procesos de diseño, planificación, implementación y evaluación de las políticas públicas en todos los niveles y en todos los ámbitos. La estrategia del Gender Mainstreaming ha sido acogida e impulsada tanto por las Naciones Unidas como por los tratados de la Unión Europea, poniendo así en la agenda política del mundo, las exigencias y el desarrollo teórico que se han elaborado desde las luchas contra la discriminación de género.

Educar en la era del postmodernismo implica el desarrollo del pensamiento crítico y la búsqueda y manejo del conocimiento, además de la toma de conciencia de la responsabilidad frente 
a los otros. No se trata de remozar la vieja educación, sino de construir una educación diferente (Lampert, 2003). Así, en el nuevo contexto ambiguo y confuso que caracteriza nuestro tiempo, la Educación juega un papel determinante porque -además de ser un medio privilegiado para la transmisión y generación del conocimiento- constituye uno de los principales mecanismos de socialización. La Educación debe levantarse de nuevo como un pilar fundamental del desarrollo humano en paz y Democracia.

Para concretar estos cambios Rodríguez (2004) señala que la educación formal, es decir, la institucional, requiere de un proyecto claro lo cual implica la gestión de la transformación desde sus bases.

Asumir la Transversalidad de Género en la Educación es plantear estrategias que desde procesos políticos posibiliten la equidad de género más allá de las aulas: en la sociedad en su más amplio sentido, impulsando el salto cualitativo hacia una igualdad y equidad real y no solo formal. Responde también al ordenamiento jurídico nacional e internacional que avala estos procesos.

Sin embargo, la implementación es un proceso complejo, con múltiples aristas que inciden en sus logros, aún cuando se asuma como estrategia institucional. Se centrará este ensayo en la Transversalidad de Género en la Educación Superior a partir de la propuesta de un modelo de implementación, focalizando las aplicaciones académicas posibles, sus fortalezas y limitaciones.

\section{La Transversalidad de Género Origen del concepto}

Posgrado y Sociedad Vol. 12 No. 1 Año. 2012
Los esfuerzos para la promoción de la mujer, la equidad de género y la igualdad entre hombres y mujeres, han pasado por diferentes momentos (UNESCO, 2009):

1. Acciones positivas focalizadas en las mujeres que generaron beneficios en un número limitado de ellas, y que no abordaban aspectos estructurales.

2. En los años 70 se reenfocan las estrategias hacia el incremento de la participación femenina en las agendas de desarrollo, esfuerzos que se incorporaban tarde en los procesos de manera tal que no se participaba en la toma de decisiones, por lo que el potencial de cambio estructural necesario para la igualdad se vio muy limitada.

3. La estrategia de transversalidad de género (Gender Mainstreaming), acogida e impulsada tanto por las Naciones Unidas como por los tratados de la Comisión Europea como una importante estrategia global para la promoción de la equidad de género al propiciar impactos estructurales que rompan desigualdades, incorporándose en los procesos de diseño, planificación, implementación $y$ evaluación de las políticas públicas en todos los niveles y en todos los ámbitos.

"Gender Mainstreaming", como una iniciativa política, surge y se desarrolla en las Conferencias Mundiales sobre la Mujer (ONU) iniciando en la Ciudad de México en 1975 y culminando (siendo adoptada ampliamente) en la IV Conferencia Mundial de la Organización de Naciones Unidas sobre 
la Mujer en Beijing (1995) y su seguimiento en la $23^{\circ}$ sesión especial de la Asamblea General (Beijing+5) en Nueva York en el año 2000 (Carney, 2004). En 1996 la Comisión Europea define este concepto y recomienda su inserción en todas las dimensiones de la toma de decisiones de cada Estado miembro; en 1997 la ONU en introduce esta estrategia en todas las políticas y programas de su sistema. ${ }^{2}$

Así, la Transversalidad de Género surge de las reflexiones, análisis y valoraciones de las prácticas y políticas realizadas para lograr la igualdad y equidad entre hombres $\mathrm{y}$ mujeres, evidenciándose que, si bien se han logrado avances importantes en este terreno, no se estaba logrando modificar los elementos o factores estructurales que configuran la desigualdad.

\section{¿Qué es Transversalidad de Género3?}

2

http://www.un.org/womenwatch/osagi/pdf/ECOSOC AC1997.2.PDF.

${ }^{3}$ La traducción al español de este término ha generado problemas de comprensión pues el propio mainstreaming se concibe de diversas formas y hay conceptos asociados (cross-cutting y target group, por ejemplo), con lo cual se producen efectos, logros y limitaciones diferentes. Esta diversidad en las aproximaciones puede deberse, en parte, a la rápida extensión del concepto, a su complejidad y a su formulación vaga en la dimensión práctica, que han permitido múltiples lecturas del mismo (Verloo, 2005, citada por Alfama y Cruells; Serra, 2004: 5).
Partimos de la comprensión de Género como una construcción simbólica, social, establecida sobre los datos biológicos de la diferencia sexual (Lamas, 1996, pp. 12-110), una especie de "filtro" cultural con el que interpretamos el mundo y una armadura con la que limitamos nuestra vida, filtrado por el lenguaje (Bordieu, 2000). Es el resultado de la producción de normas culturales sobre el comportamiento de los hombres y las mujeres, mediado por la compleja interacción de un amplio espectro de instituciones económicas, sociales, políticas y religiosas.

La transversalidad, por su parte, implica involucrar, incorporar, cruzar, estar inmerso en..., todos adjetivos que remiten a cambio y transformación al integrarse varios elementos. Esta multiplicidad de significados se refleja en la diversidad de concepciones bajo el mismo término, lo que ha generado interesantes debates.

Para Oraisón (2000, citado por Molina, 2007, p. 134) con el término "transversal", en Educación se hace alusión "a la ubicación o al espacio que se pretenden ocupen ciertos contenidos dentro de la estructura curricular de cada ciclo o nivel. Estos contenidos son concebidos como ejes que atraviesan en forma longitudinal y horizontal el currículo, de tal manera que en torno a ellos se articulan los temas de las diferentes áreas de formación".

Por su parte Munévar y Villaseñor (2005, p.53) señalan que la transversalidad puede expresarse tanto 
como temas transversales o como ejes transversales. El primero hace referencia al abordaje como contenidos que pueden ser enfocados desde distintas perspectivas disciplinarias $\mathrm{y}$ que, por lo tanto, dan posibilidad a una relación entre éstas y a una mayor conexión entre lo tratado en clase con la realidad del alumnado. Los temas, desde esta perspectiva, provocan cambios metodológicos sin necesariamente alterar los trasfondos ideológico y axiológico del proceso educativo. Los ejes transversales surgen de necesidades y demandas que la sociedad plantea a la educación y es alrededor de estos que debe organizarse toda la vida educativa, a partir de la planificación ya que no sólo "cruzan" las asignaturas y diversas áreas de trabajo, sino que señala un camino a seguir y una meta formativa de interés escolar4.

Es interesante señalar que para Munévar y Villaseñor (2005) la transversalidad es uno de los temas puestos a debate para lograr la ruptura de fronteras disciplinarias y lograr dar respuesta a los nuevos fenómenos sociales. Por ello, más que atender a contenidos cognitivos, los ejes nos plantean retos referidos a la formación integral de las personas en dirección a lograr una ciudadanía plena y, como una característica común a todos ellos, relacionando en última instancia, valores.

\footnotetext{
4 Esto implica una visión integral del currículum, abordando todas sus dimensiones $\mathrm{y}$, en este caso, particularmente el por qué del mismo, replanteándose además una estructura, propósitos y formas relacionales viejas, que no son congruentes con esta metodología.
}

Dado que, como señalamos en páginas anteriores, la Transversalidad de Género nace desde organizaciones políticas, la definición que ofrece la Comisión Europea en 1996 presenta elementos importantes (Lombardo, 2003):

“... es la integración sistemática de las situaciones, prioridades $y$ necesidades de mujeres y hombres, respectivamente, en todas las políticas $y$ actividades, con vistas a promover la igualdad entre ambos sexos, y a movilizar todas las políticas y las medidas generales con el propósito específico de alcanzar la igualdad, teniendo en cuenta, de manera activa $y$ abierta, durante la fase de planificación, sus efectos sobre las situaciones respectivas de mujeres $y$ hombres durante la ejecución, el control y la evaluación"

Sin embargo para esta misma autora la definición más aceptada, completa y detallada es la que presenta el Grupo Experto del Consejo de Europa en 1998 (citado por Lombardo, 2003):

'El mainstreaming de género es la organización (la reorganización), la mejora, el desarrollo y la evaluación de los procesos políticos, de modo que una perspectiva de igualdad de género se incorpore en todas las políticas, a todos los niveles y en todas las etapas, por los actores normalmente involucrados en la adopción de medidas políticas.'

Integrando algunas ideas de la literatura consultada (Unión Europea, 1998; Consejo de Europa, 1999; UNESCO, 2009; Lombardo, 2003; Montané, s.f.; Munévar \& Villaseñor, 2005; Alfama \& Cruells, 2009, entre 
otros), entiendo la Transversalidad de Género como:

una herramienta estratégica de transformación política (progresiva) de las estructuras sociales sobre las que se construyen y mantienen los estereotipos de género. Mediante un proceso complejo y dinámico de análisis, intervención y gestión, impulsa a los actores involucrados en la adopción de políticas públicas a que, incorporen claramente la perspectiva de género, equidad e igualdad de oportunidades entre mujeres y hombres en la agenda política dominante, reflejándose en las decisiones de todas las áreas políticas y en todos los sistemas (social, económico, político), estructuras y cultura, tanto en la esfera pública como en la privada.

Así, la Transversalidad de Género es una forma de expresión del deseo político de trasladar la equidad a la práctica social desde las políticas públicas, para incorporar nuevas visiones $\mathrm{y}$ valores en los actores sociales.

Como finalidad de la Transversalidad de Género se pueden visualizar varios aspectos:

1. incorporación de la perspectiva de género en los procesos de toma de decisiones en cada etapa del ciclo de planeamiento y programación;

2. proyección de los posibles impactos que las diferentes medidas, políticas y programas puedan tener sobre los hombres y sobre las mujeres, de

Posgrado y Sociedad Vol. 12 No. 1 Año. 2012 manera que se puedan minimizar los efectos paradójicos 5 .

3. promoción de la equidad de género y la igualdad de oportunidades para hombres y mujeres, tanto real como formal;

4. empoderamiento femenino; lo que incluye la inclusión y participación de las mujeres en instituciones $\mathrm{y}$ procesos de toma de decisiones (Lombardo, 2003:3)

5. cambio estructural: transformación de los mecanismos y procesos que reproducen estructuralmente las desigualdades (lo que implica la incidencia en políticas públicas);

6. rediseño cultural (transformación género-sensible) a través del diseño, implementación, monitoreo y evaluación de políticas y programas que permitan el logro de la equidad e igualdad en la cotidianidad y la interacción con otros

La Transversalidad de Género se fundamenta en el enfoque sistémico, en la inclusión del género como categoría

\footnotetext{
${ }^{5}$ Un ejemplo interesante es el de la reforma al Código de Trabajo costarricense propiciada por la Ley 7142, de Promoción de la Igualdad Social de la Mujer (1990): en su artículo 100 estable que cuando en una organización existieran trabajando más de 30 mujeres, el patrono deberá "acondicionar un local a propósito para que las madres amamanten sin peligro a sus hijos". En sus inicios el efecto paradójico de esta legislación fue un inicio fue el control de la contratación femenina para no requerir del cumplimiento de esta norma. Así, en lugar de modificar las condiciones de discriminación que existen en el ámbito laboral nacional para las mujeres madres, la profundizó.
} 
de análisis mediante la combinación de buenas prácticas, medidas específicas, políticas de promoción, y la inclusión de saberes de género que requieren de la integración de conocimientos interdisciplinarios (Munévar \& Villaseñor, 2005) ${ }^{6}$. Se asume como un programa político de cambio progresivo (Alfama \& Cruells, 2009:14), es decir, un proceso, dado que el modelo hegemónico actual de política en general y de políticas públicas en particular se sustenta en la exclusión de amplios sectores sociales y en unos valores androcéntricos, reconociendo así que las prácticas institucionalizadas del propio Estado generan desventajas personales y de grupo. Por lo tanto se apunta a transformar los mecanismos y procesos que reproducen estructuralmente las desigualdades lo cual requiere de modificaciones profundas en la cultura organizacional e institucional de las administraciones públicas que permitan llevar adelante estos cambios sociales.

Además, tal como Serra (2004) lo indica, la transversalidad de género genera nuevos retos organizativos orientados a la dotación de los instrumentos adecuados para conseguir que la acción pública de todas y cada

\footnotetext{
${ }^{6}$ Transdisciplinarios podría ser una perspectiva más congruente con esta propuesta pues, siguiendo la propuesta de Bunge (1995), para abordar problemas sociales es "Paso a paso, pero todos juntos", refiriéndose a la transdisciplinariedad, dado que los problemas humanos son polifacéticos y se dan en sistemas, no uno a uno.

La transdisciplinariedad es un concepto "reciente" que autores como Eric Jantsch, Piaget y Morin han trabajado en los últimos treinta años; es multirreferencial y multidimensional.
}

una de las organizaciones sea coherente en su actuación en tres sentidos:

1- Que las políticas, en toda su implementación, no generen discriminación de género.

2- Que toda su actuación aporte elementos de promoción de una mayor y creciente igualdad de género.

3- Que estos dos objetivos pasen a ser objetivos adicionales de todos los sectores de actuación y de todas sus actividades.

\section{Importancia de la \\ Transversalidad de Género en la Educación Superior}

Una educación auténtica no puede privilegiar la abstracción en el conocimiento sino que debe enseñar a contextualizar, concretar y globalizar. Esta premisa es importante para entender las propuestas de Valleys (2007) sobre la Educación Superior.

Para este autor, los dos principales propósitos académicos de la Educación Superior son la formación humana y profesional (propósito educativo) y la construcción de nuevos conocimientos (propósito de investigación), fines estrechamente relacionados pues se alimentan mutuamente, en procura del desarrollo crítico que posibilite el cambio social.

Agrega que los impactos que genera la Universidad en su cotidianidad, pueden ser agrupados en cuatro rubros:

1. Impactos de funcionamiento organizacional, pues como cualquier organización laboral, la Universidad deja huellas en la vida de su personal administrativo, docente $\mathrm{y}$ estudiantil, al ser modelo de acción. 
2. Impactos educativos directamente sobre la formación de los jóvenes y profesionales, su manera de entender e interpretar el mundo, comportarse en él y valorar ciertas cosas en su vida... Influye asimismo sobre la deontología profesional, orienta (de modo consciente o no) la definición de la ética profesional de cada disciplina y su rol social.

3. Impactos cognitivos epistemológicos dado que al orientar la producción del saber y las tecnologías, influye en la definición de lo que se reconoce socialmente como "Verdad, Ciencia, Racionalidad, Legitimidad, Utilidad, Enseñanza..." Incentiva (o no) la fragmentación y separación de los saberes al participar en la delimitación de los ámbitos de cada especialidad.

4. Impactos sociales: la Universidad es una de las instituciones con capacidad de decisión y de ejecución de medidas que afectan a toda la sociedad y su desarrollo económico, social y político, tanto de forma directa (en cuanto forma a sus profesionales y líderes) e indirecta en cuanto a que es un referente y un actor social, que puede promover (o no) el progreso, que puede crear (o no) Capital Social, vincular (o no) la educación de los estudiantes con la realidad social exterior, hacer accesible (o no) el conocimiento a todos, posibilitando (o no) el cambio en la estructura social y económicopolítica.
Edgar Morín (1999) por su parte, aporta una visión de ser humano como una unidad compleja, sistémica que, por su naturaleza, es a la vez físico, biológico, psíquico, cultural, social e histórico, y una forma de pensar que dé cuenta de que el conocimiento de las partes depende del conocimiento del todo, y que el conocimiento del todo depende del conocimiento de las partes (enfoque sistémico); así mismo, que reconozca $y$ analice los fenómenos multidimensionales en lugar de aislar, mutilando, cada una de sus dimensiones y analice las realidades que son al mismo tiempo solidarias y conflictivas (Principio Holográmico de Morin, 1998, p.99). Ello sin obviar el respeto a lo diverso, en donde al mismo tiempo se reconozca la unidad7.

Desde estos planteamientos y el referente conceptual sobre Transversalidad de Género, la incorporación de esta herramienta

\footnotetext{
${ }^{7}$ Su propuesta es el Pensamiento Complejo: es la posibilidad de reconocer los principios de incompletud y de incertidumbre en el seno de todo conocimiento, ante el cual se ha de aspirar a un saber no parcelado, no dividido, no reduccionista, evitando un conocimientoacción unidimensional y mutilante. Lograr un pensamiento multidimensional que sea capaz de concebir la complejidad de lo real, hace necesario la búsqueda de un método para "Unir lo separado" - "Articular lo que está desunido”. Para esto el autor propone siete principios (1999:98 y siguientes) que integran la enseñanza del pensamiento complejo y de la condición humana como una unidad, para que el proceso formativo esté guiado en el conocimiento ser humano, situado en el universo.
} 
estratégica en la Educación Superior implica integrar la perspectiva de género (análisis, seguimiento y evaluación) en planes, programas y proyectos académicos que generen cambios en la formulación de leyes, políticas, cultura organizacional, prácticas sociales basadas en el intercambio y medios de interacción con las comunidades para lograr la equidad de género en la educación superior (UNESCO, 2009), así como la transformación política de las estructuras sociales sobre las que se construyen y mantienen los estereotipos de género, dentro y fuera de los sistemas educativos (Munévar y Villaseñor, 2005). Implica que la perspectiva de género debe atravesar la Universidad tanto de forma horizontal como vertical (Díaz-Barriga, 2006), desde tres perspectivas: cognitiva, conductual y emocional (interjuego que da un sentido particular a su atención) $\mathrm{y}$ en todo el sistema institucional (gestión, academia, extensión o proyección, investigación).

Se derivan tres objetivos de esta definición:

1. desarrollo de una cultura organizacional y académica génerosensitiva,

2. establecer una estrategia de identidad de género institucional (a lo interno de la organización, como institución),

3. establecer una estrategia de educación de género en círculos académicos (área académica en específico).

Se espera el desarrollo de una cultura género-sensitiva que no solo provea una educación académica, sino que contribuya con el análisis de la complejidad de la dimensión de género, asegurando que esta perspectiva sea tomada en cuenta en la educación, potenciando la reflexión y el cambio. Se procura de esta forma la generación de aprendizajes relevantes para la persona y para el grupo, proveyéndoles además de herramientas conceptuales y actitudinales que les permitan situarse en el mundo con capacidad de actuar e influir en él de forma consciente y crítica.

Montané (s.f., p. 3) enfatiza que la introducción educativa de la perspectiva de género de carácter transversal impide que la discriminación se enfoque únicamente desde la situación de desventaja personal de las mujeres, hace visibles las jerarquías culturales, desnaturaliza las prácticas de trabajo jerarquizadas, desarticula los sobreentendidos de género en todos los ámbitos sociales e interroga sin pausa las formas de producción, distribución y circulación de conocimientos en la academia.

\section{Implementación}

De una manera muy sucinta podemos señalar algunos momentos en el desarrollo del proceso de implementación de la Transversalidad de Género (TG) en la Educación Superior:

Debemos partir de que una institución universitaria tiene cuatro subsistemas interactuando: gestión institucional, docencia, investigación y extensión. A través de estos, horizontal y verticalmente, la incorporación de la perspectiva de Género debe realizarse.

Se asume que los actores de la TG en la Educación Superior son todos los 
grupos interesados, tanto internos como externos, directos o indirectos: líderes, trabajadores de las diferentes áreas institucionales, estudiantes, proveedores, otras universidades, gobierno y sus ministerios relacionados, sociedad civil, entre otros.

En tanto herramienta estratégica, la TG cuenta con tres tipos de instrumentos: analíticos; formativos $\mathrm{y}$ consultivos, y participativos (Serra,
2004) y una metodología para desarrollarse con cuatro fases críticas, fundamentadas en el mejoramiento continuo a partir del monitoreo y la retroalimentación de procesos, según se muestra en la figura 1. Es importante señalar que no es un proceso lineal ni es una lista de chequeo, sino algunas ideas para su concreción.

Figura 1

Modelo de implementación de Transversalidad de Género en Educación Superior

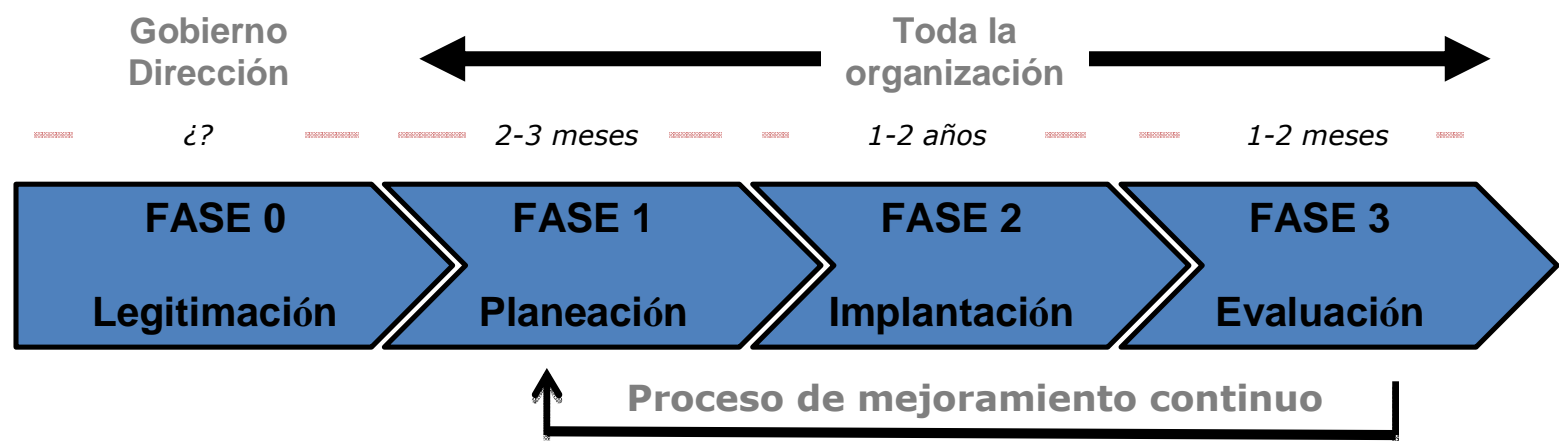

Fuente: elaboración propia a partir de UNESCO, 2009; Serra, 2004; García y Dolan, 1997;

Durán, 2008).

\section{Fases de implementación}

Fase o: Legitimación del proceso por parte de los líderes formales e implica el compromiso político de las autoridades institucionales. Implica la aceptación de la importancia de la temática y la necesidad de cambio que se traduzca en un compromiso genuino visible en acciones concretas: desde la autorización de desarrollo de la propuesta, la incorporación de TG en el Plan Estratégico Institucional de

manera que se convierta en directriz de acción a todos los niveles, y la dotación de profesionales con la calificación requerida para conformar el equipo de trabajo que lidere el desarrollo de TG en la institución (especialmente formación género-sensitiva), así como 
de los recursos necesarios para el desarrollo de la temática (económicos, tecnológicos, infraestructura).

Fase 1: Planeación génerosensitiva.

1. Requiere de la definición del equipo de trabajo facilitador y de la metodología de implementación. Señala Mirta González (2005) que este grupo especial debe estar comprometido con el diseño y evaluación de un plan de transversalidad y equidad de género, en coordinación con los diferentes actores involucrados en el proceso educativo (la comunidad y la familia por ejemplo).

Para un mejor funcionamiento e impacto institucional, es conveniente que la composición del equipo sea interdisciplinaria e interdepartamental, además con representación equitativa de ambos géneros. Dado que la claridad conceptual y del objetivo institucional son fundamentales, el equipo líder de TG debe contar con las competencias necesarias para potenciar su alcance, considerándose además los procesos necesarios de formación y capacitación en los temas que se requieran.

La responsabilidad principal es la articulación de proceso de implementación de Transversalidad de Género. Dentro de las responsabilidades del equipo base se pueden mencionar evaluaciones docentes periódicas de libros, materiales, actividades y contenidos de programas, así como del currículum oculto, con el objeto de promover la equidad.

Este grupo podrá constituirse en la base para la creación de un programa específico y permanente de Equidad de Género institucional que impulse la investigación, capacitación y formación, campañas de difusión y sensibilización del tema de género, y que tenga participación activa en el análisis y diseño de políticas institucionales desde una perspectiva género-sensitiva, además de velar por su implementación.

2. Para la definición del punto de partida y como forma de valorar los cambios que se gesten en la institución, se requiere de la investigación o diagnóstico institucional desde el análisis de género, entendido como "el abordaje de un fenómeno social a partir del enfoque diferenciado de roles, responsabilidades, necesidades y oportunidades de mujeres y hombres dentro del sistema/problema bajo análisis... Permite identificar las brechas de género y las posibles acciones para atenderlas (Pichardo, 2011).

Se requiere conocer la situación institucional actual y la normativa relacionada al tema de Género, además del análisis de género de las políticas institucionales y la identificación de oportunidades de introducción de enfoque de género en ámbitos específicos: políticas, programas, proyectos, curricula, entre otros ámbitos.

La captura, producción y tratamiento de información permitirá la identificación de las brechas, disparidades de género o prácticas discriminatorias y sistemas de opresión tanto implícitas como explícitas, por tipo y nivel de inequidad entre hombres y mujeres, facultades, estudiantes y administrativos. Pueden incluirse datos "datos duros" como matrícula, número de estudiantes por género, deserción y graduación, tiempo de duración para 
desarrollar el plan de estudios, incorporación de TG en el currículo -en cuantos, cómo se hizo-, investigación, gobierno y administración (especialmente con respecto a la asignación de recursos, instalaciones, presupuesto, información $\mathrm{y}$ comunicación, entre muchos otros).

3. Crear una visión del cambio: adonde queremos llegar.

Implica:

a. la claridad del objetivo a alcanzar: el desarrollo de una cultura organizacional y académica género-sensitiva

b. la definición de metodología de implementación lo que incluye:

objetivos y factores claves de éxito; fases de desarrollo e implementación; plan de trabajo; cronograma;

c. identificación de actores o grupos interesados que incluya los participantes por área /fase del proyecto.

4. El desarrollo de un Plan de Comunicación se constituye en una herramienta fundamental para la sensibilización y posicionamiento del tema de Género en la institución, dando a conocer los avances y logros que se vayan generando.

5. Identificación de participantes (actores o grupos interesados), tanto externos como internos, entre los cuales deben considerarse instituciones y organizaciones relacionadas a la temática, el gobierno y sus ministerios, sociedad civil, estudiantes, personal administrativo (del área financiera, planificación, administradores de centros universitarios, entro otros) y académico (escuelas y decanaturas, sistema de estudios de posgrado, extensión docente, por ejemplo), jefaturas,

Fase 2: Ejecución.

Sensibilización (Organización de capacitación y formación en Género, Transversalidad de Género, metodologías Género-sensitivas; sesiones de trabajo, reuniones, conferencias)

El establecimiento de medidas a tomar para la reducción de brechas de género y prácticas discriminatorias en la gestión universitaria y la academia, requiere del desarrollo de lineamientos, implementación y alineamiento de prácticas a partir del diseño e identificación de objetivos estratégicos y operativos y formulación de políticas y estrategias, además del desarrollo de instrumentos y metodologías de trabajo.

Guzmán (2004, p. 19) señala la conveniencia e importante de que la institución explicite en documentos oficiales su compromiso con la igualdad y la equidad de género: "Esta es una institución que favorece la igualdad de oportunidades entre mujeres $y$ hombres".

Esto por cuanto la normativa institucional es fundamental, tanto en la forma verbal de exponer las ideas como en cuanto a la revisión, análisis y elaboración de políticas congruentes se constituye en un factor clave del éxito.

Los reglamentos, por ejemplo deben usar un lenguaje que incluya tanto a hombres y mujeres. Por ejemplo 
Guzmán (2004) sugiere que en vez de utilizar "El Director será nombrado por...", se puede emplear la frase "La persona a cargo de la Dirección será nombrada por...”.

Lampert (2003) señala la importancia de considerar los tres niveles de las políticas educativas: el político-ideológico, el técnicopedagógico y el organizacional; los tres niveles deben ser considerados en este proceso.

Es fundamental la estructuración y gestión de redes de contacto (networking) internas y externas de tipo bilateral y multilateral, y el desarrollo de instrumentos relacionales informales, y la estructuración y diseño de los órganos formales que correspondan.

Cada uno de los aspectos señalados requiere del establecimiento de indicadores y mecanismos adecuados y fiables de control de procesos, que permitan realizar el monitoreo constante del avance de la implantación de TG.

Fase 3: Monitoreo y Evaluación (con perspectiva de género)

Se procura la medición de impacto de las acciones de reducción o eliminación de brechas de género $\mathrm{y}$ prácticas discriminatorias en la gestión universitaria y la academia, a partir de la valoración de los indicadores propuestos para el seguimiento del desarrollo del modelo, y mediante la definición y utilización de las herramientas necesarias para su realización (Sistema de Administración de Género -GMS- , auditoría de género, evaluación del impacto de género en programas, proyectos, curricula, por ejemplo).
A partir de la sistematización de las lecciones aprendidas en el proceso debe darse un "reciclaje" de aprendizajes que permita definir planes de mejora, implementar las mejoras identificadas y, como valore agregado y gracias al Plan de Comunicación, consolidar las mejorías y sumar adeptos al cambio

\section{La Transversalidad de Género en el área académica.}

Hasta ahora nos hemos referido a la implementación (general) de TG en la institución de Educación Superior. Vale señalar que para la atención de la TG en el área de docencia o académica, es necesario considerar seis componentes específicos que se integren en las fases del modelo propuesto:

1. Análisis de modelo pedagógico institucional: debe considerarse los conceptos institucionales de educación, estudiante, enseñanzaaprendizaje y su congruencia con la práctica, dado que orientan la gestión institucional y la entrega de la docencia.

Deben también abordarse aspectos el diseño curricular, los ejes transversales y la atención a temas relacionados con estudiantes y $\mathrm{su}$ caracterización.

2. Currículum: análisis de curricula (especialmente el oculto $^{8}$ ) e incorporación del enfoque de género en currícula, planes $\mathrm{y}$

\footnotetext{
${ }^{8}$ Munévar y Villaseñor (2005) proponen estudiar el currículum oculto en educación superior desde la investigación-acción, de manera que la comunidad educativa sea parte de su experiencia, e identifique contradicciones, las reflexione y analice de manera colectiva, buscando alternativas.
} 
programas de estudios; creación de cursos específicos, temas y contenidos sobre los estudios de género tanto a nivel de pregrado (técnico y diplomado), grado $\mathrm{y}$ posgrado de las diferentes áreas del conocimiento.

3. Investigación: Si bien esta es un área específica, aquí se considera como acción, en tanto que para el desarrollo de la docencia es fundamental la actualización y enriquecimiento que la investigación ofrece tanto para los docentes como para los estudiantes.

Uno de los problemas importantes en las instituciones educativas latinoamericanas, es su condición de "consumidoras" de investigación y no de "productoras". Se están haciendo esfuerzos importantes para variar esta situación, pero es necesario analizar el concepto de investigación en la universidad dado su contenido político y la orientación que propicia, que incluye la dotación de recursos para determinados temas y proyectos. Por esto, es importante cuestionar qué se investiga, para qué, cómo se hace, quién la y para quién se realiza.

Además de esto es necesario desarrollar términos de referencia género-sensitivos para las investigaciones e informes, considerando las condiciones e impacto diferenciado que tiene la investigación sobre mujeres y hombres ${ }^{9}$. También

\footnotetext{
9 Al respecto Queralt y Ruiz (s.f, pp. 12-13) presentan interesantes ejemplos de discriminación
}

debe propiciarse la difusión e incorporación de los hallazgos en el ámbito académico de manera que la investigación cumpla con el cometido de visibilizar, sensibilizar, generar alternativas $\mathrm{y}$, por consiguiente, gestar cambios en el entorno.

Aquí es importante enfatizar en el papel que tienen los centros expertos con que Costa Rica cuenta, tanto públicos como privados, como (y especialmente) desde las universidades con el Centro de Investigación en Estudios de la Mujer (CIEM) de la Universidad de Costa Rica; Instituto de Estudios de la Mujer (IEM) de la Universidad Nacional; Instituto de Estudios de Género de la Universidad Estatal a Distancia.

4. Material didáctico ${ }^{10}$ y mediación pedagógica: Deben evitarse los estereotipos de género $o$ de cualquier otra naturaleza11. El análisis de la perspectiva general de textos incluye imágenes, ejercicios (mediación), frecuencia de

por género en el Instituto Tecnológico de Costa Rica.

${ }^{10}$ Este en un apartado especialmente interesante por cuanto los materiales educativos y recursos didácticos transmiten valores, ideas y estilos de vida a través de los mensajes que contienen, los personajes que presentan y el lenguaje que utilizan. De esta forma se influye en la comunidad estudiantil con el tipo de referentes o modelos que se les presenta y con los cuales identificarse. (Chaves, 2010; UNESCO, 2009)

11 González (2005) recomienda utilizar el "efecto espejo" como un recurso para este proceso; consiste en cambiar los personajes: si el resultado nos suena extraño, inapropiado o risible, muy probablemente estamos ante un estereotipo. 
aparición de mujeres y hombres, cuando y cómo, con sus nombres?; naturaleza de la apariencia de los caracteres femeninos y masculinos (roles profesionales por ejemplo); ilustraciones, resultados, lenguaje inclusivo $^{12}$ (libre de sexismo), equilibrio de género en actividades de aprendizaje, texto e imágenes. Se requiere evidenciar la presencia y aportes de las mujeres equilibradamente, no como situaciones o casos excepcionales o esporádicos, destacando por igual los logros de mujeres y hombres, evitando aquellas frases $o$ expresiones que refuerzan estereotipos de género. Ninguno de los sexos aparecerá mencionado o ilustrado como superior o inferior al otro, evitando representaciones tradicionales.

Guzmán (2004) señala la importancia de que los recursos didácticos ofrezcan "modelos positivos" de mujeres ejerciendo puestos de decisión o responsabilidad: ingenieras, alcaldesas, ministras, presidentas, magistradas, además de incorporar

12 El lenguaje debe considerarse en todas las fases y niveles, pues su poder es muy grande: influye en nuestra percepción de la realidad ya que condiciona nuestro pensamiento y determina nuestra visión de mundo, a través de este se reflejan y refuerzan las desigualdades sociales y de género. En términos generales se puede hablar tanto de un lenguaje sexista como de un uso sexista del lenguaje. (Chaves, 2010). $\mathrm{Al}$ respecto Guzmán (2004, p. 6) indica que "no nombrar a las mujeres en discursos, textos e ilustraciones que hacen referencia al quehacer humano, a grupos sociales o a la sociedad, es invisibilizarlas. Esta acción es sexista porque sobrevalora lo masculino, a la vez que desvaloriza lo femenino y a las mujeres. Las desconoce como personas". ilustraciones de figuras representando autoridad o espacios de toma de decisiones políticas, empresariales o profesionales que presenten en forma equilibrada a ambos sexos.

5. Evaluación de aprendizajes, de procesos y de impacto: incluye aspectos como factores que inciden en el rendimiento de estudiantes por curso (género, lugar de procedencia, acceso a tecnología por ejemplo), acciones consecuentes,

6. Autoevaluación de Unidades Académicas periódicamente que permitan la reflexión del quehacer académico en cada una de ellas en los apartados anteriores, incluyendo aspectos de calidad en general de la enseñanza-aprendizaje y su impacto (valoración por género de efectos positivos o negativos por carrera, número de estudiantes por carrera), valoración de la igualdad de trato y participación en las aulas (presenciales 0 virtuales), valoración de las graduaciones por carrera (factores que inciden en la incorporación al mercado laboral, tipo de inserción $\mathrm{y}$ otros). Esta propuesta se visualiza en la siguiente figura 
Figura 2

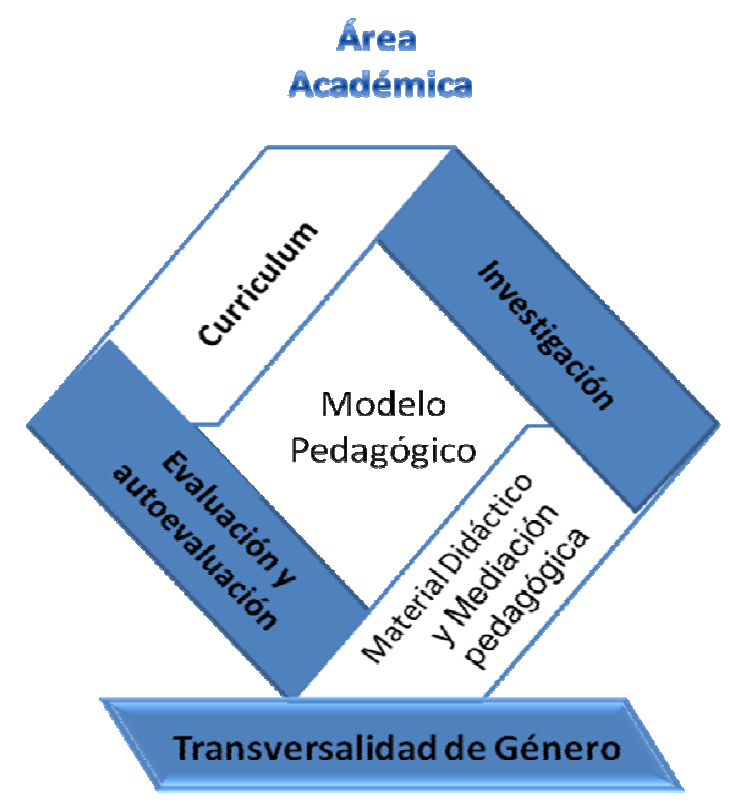

Fuente: elaboración propia

\section{Conclusiones}

La Transversalidad de Género nace como una herramienta política para el cambio estratégico en temas de sociales orientados hacia la igualdad y equidad de género.

Señala Carney (2005) que la Transversalidad de Género es una transformación global en la que sus aplicaciones reflejan las normas culturales locales $y$ las relaciones humanas, políticas y laborales ya que tiene que ser asumida por todos los actores que participan en la elaboración, adopción e implementación de medidas políticas.

Requiere de una variedad de condiciones para su concreción, entre las que podemos mencionar como factores clave de éxito las siguientes:

- voluntad (y oportunidad) política: Liderazgo legitimador (apoyo, compromiso y soporte genuino de líderes) e incorporación de directrices
- en planes estratégicos y normativa, correlación de fuerzas); - claridad conceptual y metodológica que se reflejen en las metas estratégicas,

- normativa congruente, - redes de apoyo de actores involucrados en las áreas temáticas - sensibilización y formación profesional en el conocimiento de situaciones específicas, intereses $\mathrm{y}$ necesidades particulares de hombres y mujeres (asumiendo el aprendizaje organizacional),

- $\quad$ análisis de barreras; - adecuación de los marcos político ideológicos orientando las políticas sectoriales, - elaboración de medidas que favorecen que favorecen la equidad de género,

- articulación de procesos académicos,

- buena gestión y apropiadas condiciones de implementación, 
- una gran dosis de pasión, creatividad y perseverancia.

Incluir la perspectiva de género en la educación y la formación es una tarea necesaria, pero además inevitable en el contexto actual pues aspiramos a fortalecer los derechos humanos y la democracia desde la asunción de la alteridad. En la Educación Superior su incorporación es un tema fundamental en aras de lograr más educación para todos, con equidad e igualdad, derechos básicos que rigen el funcionamiento de las sociedades democráticas.

Señala Montané (s.f.) que incorporar la TG en la educación superior es una tarea necesaria e inevitable ya que la nueva y compleja realidad precisa de nuevas miradas y habilidades complejas, necesita incorporar la mirada femenina que genere una revolución en la equidad de género y la igualdad real y formal en las oportunidades de hombres y mujeres, articulados desde los derechos fundamentales. Hay otro aspecto fundamental: Costa Rica es signataria de la Convención para la Eliminación de Todas las Formas de Discriminación contra la Mujer (CEDAW)13, lo que implica una presión adicional para atender las implicaciones de este compromiso.

Para su logro se proponen como pilares el aspecto académico, la investigación y la gestión que, integrados, potenciados y monitoreados

\footnotetext{
${ }^{13}$ Ratificada por ley No. 6968 del 2 de octubre de 1984, publicada en el Diario Oficial el 11 de enero de 1985, y depositado el instrumento, en la Secretaría de la Organización de las Naciones Unidas, el día 4 de abril de 1986.
}

pueden propiciar desarrollo de una cultura organizacional y académica género-sensitiva que se proyecte a la sociedad a través de nuestros estudiantes y profesionales.

Hay dificultades relacionadas a la transversalización de género en las universidades. Una de estas es la interpretación de que solamente se trata de abrir espacios de participación para las mujeres o que se desarrollen programas específicos para ellas; sin embargo, a pesar de la gran importancia de la participación y acceso a espacios tradicionalmente cerrados o limitados para las mujeres, se requiere que el espacio ofrezca recursos, o estrategias para que este sector tenga opciones de avanzar en las estructuras sociales. Es por esto necesario alertar sobre el riesgo de que la TG sea usada o comprendida como una sustitución de políticas específicas o que la falta de claridad conceptual incida en esfuerzos desintegrados e incongruentes, impactando los objetivos estratégicos que pueden diluirse o tergiversarse. La Transversalidad de Género no elimina la necesidad de elaborar políticas y programas específicos para las mujeres, así como legislación positiva a su favor; tampoco elimina la necesidad de unidades o coordinaciones para la atención de aspectos de género.

Otro aspecto es el que la evaluar su impacto no es sencillo y se requiere un proceso de sensibilización $\mathrm{y}$ formación muy importante.

Sin embargo desde las instituciones de educación superior, como actores y gestores de cambio social, no podemos (ni debemos) desvincularnos de temas 


\section{La Transversalidad de Género en la Educación Superior: propuesta de un modelo de implementación}

fundamentales como este pues evidencia la firme voluntad de lograr justicia social y el reconocimiento de derechos. El compromiso legal y ético es motor de transformaciones que los profesionales de nuestro país deben liderar desde la equidad y la igualdad de géneros.

\section{Referencias}

Acker, S. (1995). Género y educación. Reflexiones sociológicas sobre mujeres, enseñanza y feminismo. Pp. 9155. España: Narcea.

Acaso, M; Nuere, S. (2005). El currículum oculto visual: aprender a obedecer a través de la imagen. Recuperado de http://revistas.ucm.es/bba/11315598/articulos/ARISo505110207A.PDF

Aguerrondo, I. (1999). El nuevo paradigma de la Educación para el siglo. OEI. Accesible en http://www.campus-oei.org/administracion/aguerrondo.htm

Aguerrondo, Inés (2010). Innovación en contextos educativos complejos: el reto de la generalización. Conferencia presentada en el Congreso Internacional de Administración de la Educación, Escuela de Administración Educativa de la Universidad de Costa Rica.

Alfama, E.; Cruells, M. (2009). ¿Hacia unas políticas públicas más inclusivas?. Reflexión sobre los avances y límites en base a las Políticas de igualdad de género. Ponencia presentada en el IX Congreso de Ciencia Política y Administración: "Repensar la democracia: inclusión y diversidad". Málaga. Recuperado de

http://www.aecpa.es/archivos/congresos/congreso_09/grupos-trabajo/areao6/GTo3/o1.pdf

Araya Umaña, Sandra (2001). Cambian los discursos: ¿Cambios en las prácticas?. Revista La Ventana, núm. 14 / 2001. págs 159-201. Recuperado de

http://148.202.18.157/sitios/publicacionesite/pperiod/laventan/Ventana14/14-5.pdf

Araya Umaña, Sandra (2003). Caminos recorridos por las políticas educativas de género. Revista Electrónica Actualidades investigativas en Educación. Julio-Diciembre, Vol 3, Núm 2, Año 2003. Recuperado de http://redalyc.uaemex.mx/pdf/447/44730202.pdf

Association of Development or Education in Africa (2006). A Toollkit for Mainstreaming Gender in Higher Education in Africa. Accra: Association of African Universities. Recuperado de http://www.aau.org/wghe/gender/toolkit/Toolkit-module1.pdf

Bacchi, C; Eveline, J. (2010). Mainstreaming politics: gendering practices and feminist theory. University of Adelaide

Press.

Recuperado http://www.adelaide.edu.au/press/titles/mainstreaming/Mainstreaming-Ebook-final.pdf

Binder, Beate; Pache, Ilona (2008). Gender - Knowledge - Participation. Professional Orientation and Career Paths of Gender Studies Graduates. Berlin Universität. Recuperado de http://www.gender.huberlin.de/w/files/ztgbulletintexte34/8binder_pache.pdf

Bourdieu, Pierre (2000). La dominación masculina. Barcelona: Anagrama. Título del original en francés La domination masculine (1998).

Bunge, M (1995). Sistemas sociales y filosofía. Buenos Aires: Editorial Sudamericana.

Carney, Gemma (2005). Data, anecdote and metaphor in Gender Equality Policy-making: merging "intelectual and real world mainstreaming". Granada. Recuperado de http://eis.bris.ac.uk/ potfc/Granada/Papers/Carney.pdf

Carney, Gemma (2003). ¿Communicating or Just Talking? Gender Mainstreaming and the Communication of Global Feminism. Women and Language Journal, vol. XXVI, Iss, 1, Urbana. Primavera, 2003, p. 52.

Carney, Gemma (2004). Gender and Childcare: Creating a space for 'womenandchildren' in Irish policymaking. Dublin. Recuperado de www.ispa.ie/documents/170904gcarney.doc

Chaves, Rocío. Comunicación personal. 4 de Agosto 2010. San José: UNED

Chaves, Rocío (s.f). Propuesta de incorporación de la perspectiva de género en el proceso de diseño curricular en la UNED. San José: UNED.

Council of Europe (2004). Promoting Gender Mainstreaming in Schools. Recuperado de http://www.coe.int/t/dghl/standardsetting/equality/o3themes/gender-mainstreaming/EG-SGS(2004)RAPFIN_en.pdf 
Consejo de Europa (1999). Mainstreaming de género. Marco conceptual, metodología y presentación de "buenas prácticas". Informe final de las actividades del Grupo de especialistas en Mainstreaming. Instituto de la Mujer, Ministerio de Trabajo y Asuntos Sociales, Serie documentos, Madrid, 1999.

Díaz Barriga, Ángel (2006). La educación en valores: Avatares del currículum formal, oculto y los temas transversales. Universidad Nacional Autónoma de México. Recuperado de

http://redalyc.uaemex.mx/src/inicio/ArtPdfRed.jsp?iCve=15508101

Durán, M. (2008). La Administración por Valores: una metodología humanista de cambio cultural en la empresa. Revista de Ciencias Económicas. Universidad de Costa Rica Volumen XXVI número 2, juliodiciembre 2008

Facio, A (1996). Cuando el género suena cambios trae (una metodología para el análisis de género del fenómeno legal). San José - Costa Rica: ILANUD.

Greed C. (2004). Gender Mainstreaming. Women and Environments International Magazine, Núm. 62/63, Toronto, primavera, 2004, p. 23.

González, M. (2005). El sexismo en la Educación: una barrera a superar. En Género, valores y sociedad. Una propuesta desde Iberoamérica. Fernández, L. (coordinadora). España: Octaedro

Guzmán, L; Letendre, A. (2003). Género y educación en Costa Rica. UNESCO. Recuperado de http://unesdoc.unesco.org/images/o014/001467/146775s.pdf

Humboldt Universität zu Berlin. 5th European Conference on Gender Equality in Higher Education. Results and Recommendations. Berlin: August 28 - 31, 2007. Recuperado de http://www2.hu-berlin.de/eqberlin2007/Brosch\%FCre\%20Empfehlungen\%20englisch.pdf

Kahlert , H. (2009). Changing the Gender Order. 6th European Conference on Gender Equality in Higher Education, 5.-8. August 2009 in Stockholm. Recuperado de http://budrichjournals.de/index.php/gender/article/view/3427/2952

Ketterer, L. (2008). La transversalización de género en la educación: ¿Qué pasa en las escuelas de Galvarino, la capital indígena de la región de La Araucanía? En Revista La Aljaba. Segunda época, Volumen XII, 2008 21: Universidad de La Frontera.

Lagarde, M. (1992). La condición del Género Femenino. Poder y Género. Material del curso "Identidad de Género". Managua

Lamas, M. (1996). Uso, dificultades y posibilidades de la categoría de género. En: Lamas, M (comp.) El género: la construcción cultural de la diferencia sexual. PUEG/UNAM, México. pp. 327-364.

Lampert, E. (2003). Educación: visión panorámica mundial y perspectivas para el siglo XXI. Revista Perfiles Educativos 2003; XXV, No 101: 7-22. UNAM, México

Lombardo, E. (2003). El Mainstreaming de Género en la Unión Europea. Aequalitas. Revista Jurídica de Igualdad de Oportunidades entre Mujeres y Hombres, vol. 10-15, Mayo-Diciembre 2003, pp. 6-11. Recuperado de http://www.sernam.cl/pmg/archivos_2007/pdf/Art_MainstreamingUE.pdf

Martínez, M. (2007). Conceptualización de la Transdisciplinariedad. Caracas. Recuperado de www.revistapolis.cl/polis\%2ofinal/16/doc/marti.doc

Martínez, M. (s.f.). Transdisciplinariedad y Lógica Dialéctica: Un enfoque para la complejidad del mundo actual. Caracas. Recuperado de http://prof.usb.ve/miguelm/transdiscylogicadialectica.html

Molina, D. (2007). Ejes transversales en el currículo universitario: experiencia en la carrera de derecho. Ciências \& Cognição 2007; Vol 10: 132-146. Recuperado de

http://www.cienciasecognicao.org/pdf/v10/m317153.pdf

Montané, A. (s.f.). Perspectiva de Género: educación, formación y empleabilidad. Pensamientos y políticas. Ponencia presentada en las Jornadas CONTA CON ELAS. Universitat de Barcelona. Recuperado de

http://www.contaconelas.org/materiales_files/4._A_transversalidade_..._Montane.pdf

Morin, E. (1999). La cabeza bien puesta. Buenos Aires: Nueva Visión.

Morín, E. (1999). Los siete saberes necesarios para la educación del futuro. UNESCO. Recuperado de

http://unesdoc.unesco.org/images/o011/o01177/117740so.pdf

Munévar, D \& Villaseñor, M. (2005). Transversalidad de género. Una estrategia para el uso político-educativo de sus saberes. La Ventana: Revista de Estudios de Género. Guadalajara, México, No. 21, 2005, pp. 4468. Recuperado de http://redalyc.uaemex.mx/redalyc/pdf/884/88402102.pdf

Organización de Naciones Unidas. Documentos de la Cuarta Conferencia Mundial sobre la Mujer (Beijing, 1995). Recuperado de http://www.cinu.org.mx/biblioteca/documentos/dh/ConfBeijing1995.htm

Pascual, M. (1996). Los programas de diversificación curricular desde la transversalidad. Revista Enseñanza, 14, 1996, pp. 157-18o. Universidad de Oviedo. Recuperado de http://campus.usal.es/ revistas_trabajo/index.php/o212-5374/article/view/4027

Pichardo, A. (2011). Taller: Transversalidad de género y masculinidades. San José: Instituto de Estudios de Género, Universidad Estatal a Distancia. 


\section{La Transversalidad de Género en la Educación Superior: propuesta de un modelo de implementación}

Queralt, L. \& Ruiz, A. (s.f.). Perspectiva de género como eje transversal del quehacer academico e institucional: el caso del Instituto Tecnológico de Costa Rica. Recuperado de http://www.tec.cr/sitios/Docencia/ceda/Boletin_CEDA/boletin_15/Articulo_genero.pdf

Redón, S. (2007). Significados de la transversalidad en el currículum: Un estudio de caso. Revista Iberoamericana de Educación, n. ${ }^{0} 43 / 2-10$ de junio de 2007 . Recuperado de http://www.rieoei.org/deloslectores/1675Pantoja.pdf

Salinas, J. (1997). Nuevos ambientes de aprendizaje para una sociedad de la información. Revista Pensamiento Educativo. PUC Chile. 20, 81-104. Recuperado de http://tecnologiaedu.us.es/formaytrabajo/Documentos/lin7sal.pdf

Salomone, R. (2007). Igualdad y diferencia. La cuestión de la equidad de género en la educación. Revista Española de Pedagogía, año LXV, $\mathrm{n}^{\circ}$ 238, pp. 433-446.

Serra, A. (2004). La gestión transversal: expectativas y resultados. Ponencia presentada en el IX Congreso Internacional del CLAD sobre la Reforma del Estado y de la Administración Pública, Madrid, España, 2 - 5 Nov. 2004. $\quad$ Recuperado de http://www.hacienda.go.cr/centro/datos/Articulo/La\%20gesti\%C3\%B3n\%20transversal.pdf

Subirats, M. (1999). Género y escuela. En Lomas, C (comp.). ¿Iguales o diferentes? Género, diferencia sexual, lenguaje y educación. España: Paidós pp. 19-32

Naciones Unidas. UN Womenwatch. Recuperado de http://www.un.org/womenwatch/

UNESCO. Gender in Education. Institutionalizing Gender Mainstreaming. Recuperado de http://www.unescobkk.org/education/appeal/programme-themes/gender/themes/institutionalizinggender-mainstreaming/

UNESCO (2002). Women and management in higher education. A good practice handbook. Paris. Recuperado de http://www.unesco.org/education/pdf/singh.pdf

UNESCO (2009).Toolkit: Promoting Gender Equality in Education. 4th Edition, Bangkok: UNESCO Bangkok, 2009.

Valdés B., A. (2001). Seguimiento de acuerdos internacionales en educación y género: Nuevos aprendizajes de ciudadanía activa. Presentado en el panel: Sociedad civil, gobierno y educación en América Latina: beneficiadas/os y excluidas/os en la pugna distributiva. Red de Educación entre Mujeres de América Latina-REPEM, LASA 2001, Washington, 6-8 de setiembre de 2001. Recuperado de http://lasa.international.pitt.edu/Lasa2001/ValdesBarrientosAlejandra.pdf

Vallaeys, François (2007). Material del curso ¿Cómo enseñar Ética, Capital Social y Desarrollo en la Universidad? Estrategias de RSU. Módulo 3: Estrategias Pedagógicas: Ética Desde el Aula. OEA/BID 
María Marta Durán

Nota acerca del autor

María Marta Durán

Escuela de Ciencias de la Administración, Universidad Estatal a Distancia.

Correo electrónico: mduranr@uned.ac.cr 